\title{
Formation of 1-octen-3-ol from Aspergillus flavus conidia is accelerated after disruption of cells independently of Ppo oxygenases, and is not a main cause of inhibition of germination
}

Eight-carbon (C8) volatiles, such as 1-octen-3-ol, are ubiquitous among fungi. They are the volatiles critical for aroma and flavor of fungi, and assumed to be signals controlling germination of several fungi. In this study, we found that intact Aspergillus flavus conidia scarcely synthesized C8 volatiles but repeated freeze-thaw treatment that made the cell membrane permeable promoted $(R)$-1-octen-3-ol formation. Loss or down regulation of any one of five fatty acid oxygenases (PpoA, PpoB, PpoC, PpoD or lipoxygenase) hypothesized contribute to 1-octen-3-ol formation had little impact on production of this volatile. This suggested that none of the oxygenases were directly involved in the formation of 1-octen-3-ol or that compensatory pathways exist in the fungus. Germination of the conidia was markedly inhibited at high density $\left(1.0 \times 10^{9}\right.$ spores $\left.\mathrm{mL}^{-1}\right)$. It has been postulated that 1 -octen-3-ol is an autoinhibitor suppressing conidia germination at high density. 1-Octen-3-ol at concentration of no less than $10 \mathrm{mM}$ was needed to suppress the germination while the concentration of 1-octen-3-ol in the suspension at $1.0 \times 10^{9} \mathrm{~mL}^{-1}$ was under the detection limit $(<1 \mu \mathrm{M})$. Thus, 1-octen-3-ol was not the principal component responsible for inhibition of germination. Instead, it was evident that the other heat-labile factor(s) suppressed conidial germination. 
1 Kana Miyamoto $^{1}$, Tomoko Murakami ${ }^{1}$, Pattana Kakumyan ${ }^{1,}$, Nancy P. Keller ${ }^{2}$, Kenji Matsui ${ }^{1, *}$

$2{ }^{1}$ Department of Biological Chemistry, Faculty of Agriculture and the Department of Applied

3 Molecular Bioscience, Graduate School of Medicine, Yamaguchi University, Yamaguchi 753-

48515 , Japan

$5 \quad{ }^{2}$ Departments of Bacteriology and Medical Microbiology/Immunology, University of Wisconsin-

6 Madison, Madison, Wisconsin, USA

$7 \quad *$ Corresponding author.

8 Kenji Matsui,

9 Department of Biological Chemistry, Faculty of Agriculture and the Department of Applied

10 Molecular Bioscience, Graduate School of Medicine, Yamaguchi University, Yamaguchi 753-

118515 , Japan.

12 Tel: +81 (83) 933-5850; Fax: +81 (83) 933-5820; E-mail: matsui@yamaguchi-u.ac.jp

$13 \S$ Present address: School of Science, Mae Fah Luang University, Chiang Rai, Thailand 
14 Names, telephone numbers, and e-mail addresses for all authors:

15 Kana Miyamoto, +81-83-933-5850,s037uh@yamaguchi-u.ac.jp

16 Tomoko Murakami, +81-83-933-5850, t048uh@yamaguchi-u.ac.jp

17 Pattana Kakumyan,+66-53916780, pattana.kak@mfu.ac.th

18 Nancy P. Keller, +1-608 262-9795, npkeller@wisc.edu

19 Kenji Matsui, +81-83-933-5850, matsui@yamaguchi-u.ac.jp 
20 Subjects:

21 Keywords: Aspergillus flavus, Conidia, Carbon-eight volatiles, 1-Octen-3-ol, Fatty acid

22 oxygenases, Germination, Spore

23 Abbreviations: C8, carbon-eight; SPME, solid phase microextraction; Ppo, Psi-factor producing

24 oxygenase; LOX, lipoxygenase 


\section{INTRODUCTION}

Eight-carbon (C8) volatiles, such as $(R)-(-)-1-$ octen-3-ol, are found almost ubiquitously

among fungi, and they are the characteristic of the fungal aroma (Combet et al., 2006). In fungi,

they attract flies and mosquitoes, and in some fungi, they repel fungivory (Combet et al., 2006;

Brodhun \& Feussner, 2011). Interestingly, 1-octen-3-ol reduces dopamine levels and causes dopamine neuron degeneration in Drosophila melanogaster (Inamdar et al., 2013). induced by C8 volatiles (Nemcovic et al., 2008). On the contrary, it was postulated that 1-octen3-ol was a volatile autoinhibitor inhibiting unprofitable germination of Penicillium paneum conidia under harsh conditions, such as in highly crowded environments where competition for limited resources would be expected (Chitarra et al., 2004). The ability of C8 volatiles to regulate conidiation and germination of conidia was also reported with Aspergillus nidulans (Herrero-

37 Garcia et al., 2011) and Lecanicillium fungicola (Berendsen et al., 2013). Because of these

38 findings, it has been assumed that $\mathrm{C} 8$ volatiles, especially 1-octen-3-ol, perform signaling

39 functions; however, no conclusive evidence supporting this hypothesis has been provided. 
41 acid (S)-10-hydroperoxide (HPO) and subsequent cleavage reaction to form $(R)$-1-octen-3-ol and

42 10-oxo-(E)-8-decenoic acid in mushrooms (Agaricus bisporus) (Wurzenberger \& Grosch, 1984).

43 However, the enzyme(s) involved in this pathway has not been identified yet. A group of fatty

44 acid dioxygenases called Ppos [psi (precocious sexual inducer)-producing oxygenases] has been

45 identified in fungi (Brodhun \& Feussner, 2011). Aspergillus nidulans has three Ppos (PpoA,

46 PpoB, and PpoC), and studies on the deletion mutants indicated that all the three Ppo enzymes

47 are involved in Psi factor production (Tsitsigiannis et al., 2005). Recombinant $A$. nidulans PpoC

48 showed an activity to form (R)-10-HPO of linoleic acid, and a portion of the HPO was further

49 converted into $\mathrm{C} 8$ volatiles, such as 2-octen-1-ol, 2-octenal, 3-octanone and 1-octen-3-ol

50 probably through non-enzymatic chemical fragmentation (Brodhun et al, 2010). Many fungi also

51 have another type of fatty acid oxygenase, lipoxygenase (LOX) (Brodhun \& Feussner, 2011).

52 Ppos and LOXs are candidates for the enzyme involved in C8 volatile formation in fungi.

54 conidia of $A$. flavus. A. flavus is an opportunistic pathogen of crops causing highly problematic

55 infections because of aflatoxin contamination of seeds. A. flavus has four genes encoding Ppos,

56 namely, ppoA, ppoB, ppoC, and ppoD, and one gene encoding LOX (Brown et al., 2009). We

57 examined the formation of $\mathrm{C} 8$ volatiles in mutant lines of these fatty acid oxygenases. 
58 Furthermore, we quantified the amount of 1-octen-3-ol produced by conidia, and found that the

59 endogenous amount was too low to be accountable for inhibition of germination of conidia.

\section{MATERIALS AND METHODS}

61 Strains and culture conditions

Wild type A. flavus strain, NRRL 3357, was used in this study. The ppo deletion strains,

$63 \triangle p p o A, \triangle p p o C$, and $\triangle p p o D$, and the LOX deletion strain, $\Delta l o x$, prepared by homologous recombination in the pyrG strain NRRL 3357.5, and the RNAi mutant IRT4, depleted in expression of lox and all 4 ppo genes including $p p o B$ were also used (Brown et al., 2009). $A$. nidulans (NRRL 1092) was obtained from Japan Collection of Microorganisms at Riken

67 Bioresource Center. All strains were grown at $29^{\circ} \mathrm{C}$ on glucose minimal media (GMM) adjusted

68 at $\mathrm{pH} 6.5$ (Shimizu \& Keller, 2001) unless otherwise indicated. Conidia suspensions were

69 obtained from surface cultures incubated for 1 week by adding $10 \mathrm{~mL}$ of distilled water

70 containing $0.02 \%(\mathrm{w} / \mathrm{v})$ Tween 20 and by gentle mechanical removal with a sterile glass rod. The

71 suspension was filtered through Miracloth (Calbiochem, La Jolla, CA). 
Volatile analysis

For sensitive detection of volatiles a solid phase microextraction (SPME)-GC/MS

74 method was used, while for accurate quantification the volatiles were extracted with organic solvent for GC/MS analysis.

77 Flex (Supelco, Bellefonte, PA) was used. After incubating $300 \mu \mathrm{L}$ of $1.0 \times 10^{9} \mathrm{~mL}^{-1}$ conidial

78 suspension in $\mathrm{GMM}$ for $9 \mathrm{~h}$ at $29^{\circ} \mathrm{C}$ in a glass vial $(20 \mathrm{~mL})$, the vial was tightly capped. A portion

79 of vials was kept at $-20^{\circ} \mathrm{C}$ for $1 \mathrm{~h}$ and thawed at $30^{\circ} \mathrm{C}$ for $15 \mathrm{~min}$. The fiber was exposed to the

80 headspace of the vial at $22^{\circ} \mathrm{C}$ for $30 \mathrm{~min}$. Afterward, the fiber was transferred to an injection port

81 of GC/MS (QP-5050, Shimadzu, Kyoto, Japan) equipped with a $0.25 \mathrm{~mm} \square 30 \mathrm{~m}$ Stabiliwax

82 column (Restek, Bellefonte, PA, USA), where compounds were desorbed at $200^{\circ} \mathrm{C}$ for $1 \mathrm{~min}$. The

83 column temperature was $40^{\circ} \mathrm{C}(5 \mathrm{~min})$ to $200^{\circ} \mathrm{C}(2 \mathrm{~min})$ at $5^{\circ} \mathrm{C} \mathrm{min}^{-1}$. The carrier gas $(\mathrm{He})$ was at

$841 \mathrm{~mL} \mathrm{~min}^{-1}$. The mass detector was operated in the electron impact mode with ionization energy

85 of $70 \mathrm{eV}$. To identify each compound, we used retention indices and MS profiles of

86 corresponding authentic specimens. 
88 chloroform/methanol $(1 / 2, \mathrm{v} / \mathrm{v})$ containing $5 \mathrm{ng} \mathrm{mL}^{-1}$ nonanyl acetate (as an internal standard).

89 Thereafter, $0.4 \mathrm{~mL}$ chloroform and $0.75 \mathrm{~mL}$ of $1 \%(\mathrm{w} / \mathrm{v}) \mathrm{KCl}$ were added into the mixture,

90 vortexed, and centrifuged at $1000 \mathrm{rpm}$ for $10 \mathrm{~min}$. The organic layer was collected, and directly

91 served to GC-MS analysis under the condition shown above. For resolution of enantiomers of 1-

92 octen-3-ol, Alpha DEX 120 fused silica capillary column ( $0.25 \mathrm{~mm}$ x $30 \mathrm{~m}$, Supelco) was used at

93 a constant column temperature of $75^{\circ} \mathrm{C}$. Racemic 1-octen-3-ol was purchased from Alfa Aesar

94 (Lancashire, UK). Its enantiomers were from Acros Organics (Geel, Belgium). Quantification

95 was done with a calibration curve constructed with pure 1-octen-3-ol in GMM. The detection

96 limit of 1-octen-3-ol was $1 \mu \mathrm{M}$ with the signal to noise ratio more than 10 . Heat inactivation was

97 carried out by immersing the conidial suspension in a tightly sealed tube into boiling water for 10

$98 \mathrm{~min}$.

99 Germination of conidia

100

The density of conidia was adjusted to be $1.0 \times 10^{6}$ to $1.0 \times 10^{9} \mathrm{~mL}^{-1}$ in GMM

101 containing $0.1 \%$ agar. The aqueous solution of 1-octen-3-ol was mixed with the conidial

102 suspension when needed. The suspension $(200 \mu \mathrm{L})$ was spread on a glass slide $(26 \times 76 \mathrm{~mm})$, and

103 incubated at $29^{\circ} \mathrm{C}$ for $9 \mathrm{~h}$ in a humidified closed container. During incubation, the $\mathrm{pH}$ of medium 
104 did not change substantially. The conidia with the protrusion of a length longer than the diameter

105 of conidia were counted as germinated. The length of hyphae was determined with an ImageJ

106 software (http://rsbweb.nih.gov/ij/). Damaged or dead cells were stained with Evans Blue (Wako

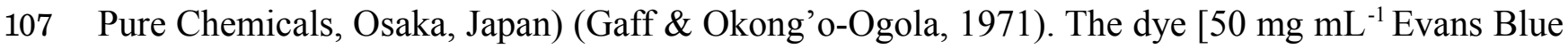

108 in $10 \%(\mathrm{w} / \mathrm{v})$ Tween $20,50 \mu \mathrm{L}$ ] was mixed with the equal volume of conidial suspension,

109 incubated for $30 \mathrm{~min}$, then, diluted four fold with distilled water for observation.

110 Glucose content

111 A. flavus conidia were incubated in $\mathrm{GMM}$ for $9 \mathrm{~h}$ at $29^{\circ} \mathrm{C}$ at $1.0 \times 10^{9} \mathrm{~mL}^{-1}$, and the

112 medium was cleared with a membrane filter (cellulose acetate $0.5 \mu \mathrm{m}$, Advantec, Tokyo, Japan).

113 Glucose content in the medium was determined with a HPLC system equipped with GL-C610H

114 column (10.7 mm i.d. x 300 mm, Hitachi High-Technologies Co., Tokyo, Japan). The mobile

115 phase was water at $0.3 \mathrm{~mL} \mathrm{~min}^{-1}$, and detection was done with a refractive index detector (L-

116 2490, Hitachi High-Technologies).

117 Evaluation of an autoinhibiting factor

118 In order to avoid the effect caused by deprivation of glucose in the medium after 
119 incubating conidia in GMM, the glucose content in the cleared medium was adjusted to $1 \%$ (w/v)

120 by using the glucose content determined as above. A portion was heat-treated in boiling water for

12110 min. Freshly prepared A. flavus conidia were suspended with fresh GMM, with the cleared,

122 spent medium, or with the heat-treated cleared, spent medium at $1.0 \times 10^{6} \mathrm{~mL}^{-1}$, and incubated for

$1239 \mathrm{~h}$ at $29^{\circ} \mathrm{C}$ as described above to see the germination.

124 Statistical analysis

125

Data obtained with at least in triplicate were analyzed using Excel-Toukei 2012 (SSRI,

126 Tokyo, Japan). Germination of conidia was calculated using Kruskal-Wallis one-way ANOVA.

127 Significant differences in their distribution at $P<0.05$ are shown with different letters. The

128 amounts of chemicals were calculated using Tukey multiple comparison test. Mean values with

129 different letters are significant at $P<0.05$.

130 RESULTS

131 Formation of carbon eight volatile compounds from $A$. flavus conidia

$132 \quad$ Volatiles were scarcely formed from intact $A$. flavus (NRRL 3357) conidia suspended in

$133 \mathrm{GMM}$ at $1.0 \times 10^{9} \mathrm{~mL}^{-1}$ (Fig. 1) and not detected in the headspace of conidiating A. flavus 
134 mycelium grown on GMM agar plate. On the contrary, volatiles were released when the

135 suspension of conidia were treated with a freeze-thaw cycle. Among the C8 compounds, 1-octen-

136 3-ol was most abundant, followed by 3-octanone, 2-octen-1-ol, and 1-octen-3-one (Fig. 1).

137 Chiral-phase GC analyses indicated that $A$. flavus conidia formed $(R)-(-)-1-$ octen-3-ol with an

138 optical purity of $>99 \%$ enantiomeric excess (Fig. 1, inset). When A. flavus conidia were treated

139 with repeating freeze-thaw cycles, the amount of 1-octen-3-ol positively correlated with

140 increased number of cycles (Fig. 2A). Evans Blue staining of the conidia indicated that the

141 freeze-thaw treatment destroyed the plasma membrane and made them permeable to the pigment

142 (Fig. 2C). The number of Evans Blue-positive cells roughly correlated with numbers of

143 ungerminated cells (Fig. 2B) and with the amount of 1-octen-3-ol (Fig. 2A). Formation of 1-

144 octen-3-ol was completely suppressed when the freeze-thaw treatment was carried out after heat-

145 treatment (in boiling water for $10 \mathrm{~min}$ ) on conidia.

146 Formation of 1-octen-3-ol from A. flavus mutants

148 formation, we quantified the volatile formed from conidia of deletion mutants $\triangle p p o A, \triangle p p o C$,

$149 \Delta p p o D$, and $\Delta l o x$ (Brown et al., 2009). IRT4 that was deficient in expression of all the five 
oxygenases ( $\triangle p p o D ; p p o A,-B$, and $-C$; and lox IRT) (Brown et al., 2009) was also used. As with

151 the wild type spores, substantial amounts of 1-octen-3-ol were only formed after the freeze-thaw

152 treatment of the mutant conidia. The amount of 1-octen-3-ol in each strain showed no significant

153 difference from that in the wild type strain (Table 1). The profiles of the other C8 volatiles

154 detected with SPME-GC/MS were also similar among all strains.

155 Effect of 1-octen-3-ol on germination of conidia

156 When conidia of $A$. flavus were suspended in GMM liquid medium at $1.0 \times 10^{6} \mathrm{~mL}^{-1}$,

157 more than $90 \%$ germinated after $9 \mathrm{~h}$ at $29^{\circ} \mathrm{C}$ (Fig. $3 \mathrm{~A}$ ). The germination rate decreased dependent

158 on spore density, and at $1.0 \times 10^{9}$ spores $/ \mathrm{mL}^{-1}$ germination was significantly inhibited. We also

159 examined germination inhibition with $A$. nidulans under the same experimental condition, and

160 obtained almost the same results (Supplemental Fig. S1). With both species, vigorous

161 germination of conidia was restored when conidia at $1.0 \times 10^{9} \mathrm{~mL}^{-1}$ incubated for $9 \mathrm{~h}$ at $29^{\circ} \mathrm{C}$

162 were diluted to $1.0 \times 10^{6} \mathrm{~mL}^{-1}$ with fresh GMM.

Next, we added various amounts of 1-octen-3-ol to the conidial suspension set at $1.0 \mathrm{x}$

$16410^{6} \mathrm{~mL}^{-1}$. Germination was not affected when the concentration of 1-octen-3-ol was below $1 \mathrm{mM}$.

165 At $10 \mathrm{mM}$ germination was slightly inhibited, and a marked inhibition was observed at $100 \mathrm{mM}$ 
166 (Fig. 3B). The germination of $A$. nidulans conidia was also inhibited with 1-octen-3-ol at concentration no less than $10 \mathrm{mM}$ (Supplemental Fig. S1).

168 Effect of glucose and $0.08 \%$ after $9 \mathrm{~h}$-incubation of the conidia at the density of $1 \times 10^{8} \mathrm{~mL}^{-1}$ and $1 \times 10^{9} \mathrm{~mL}^{-1}$, respectively. Therefore, we anticipated that availability of glucose in the medium was accountable for decreased germination. In order to examine this possibility, the conidia were 173 suspended at $2.5 \times 10^{5}$ and $2.5 \times 10^{8} \mathrm{~mL}^{-1}$ in GMM containing 0 to $4 \%$ glucose, and their germination rate was examined (Fig. 4). Without glucose, the conidia showed poor germination at both the densities, but the germination rate at high density was significantly lower than that at low density. Addition of glucose to the media efficiently promoted germination, and at $2.5 \times 10^{5}$ $\mathrm{mL}^{-1}$ addition of $1 \%$ glucose efficiently enhanced the germination rate to more than $95 \%$. At

178 higher density of conidia $\left(2.5 \times 10^{8} \mathrm{~mL}^{-1}\right)$, the germination rate increased constantly as increasing 179 the initial concentration of glucose, but the rate was still $40 \%$ even with $4 \%$ glucose. 
to incubate the conidia at $1.0 \times 10^{9} \mathrm{~mL}^{-1}$ for $9 \mathrm{~h}$, adjusted its glucose concentration to $1 \%$, and

184 suspended the conidia with the spent medium at $1.0 \times 10^{6} \mathrm{~mL}^{-1}$. The germination of conidia was significantly suppressed, and only $40 \%$ of them germinated (Fig. 5). When the spent medium was heated with boiling water for $10 \mathrm{~min}$, the germination of conidia recovered, therefore, the factor that suppressed germination was heat-labile.

\section{DISCUSSION} tissues/cells of fungi or are lacking accessibility to the substrates (linoleic acid and oxygen), perhaps due to localization of enzyme and substrates in different intracellular compartments.

196 Compartmentalization would be destroyed during loss of cell wall/membrane integrity thus 
197

198

199

200

201

202

203

204

205

206

207

208

209

210

211

allowing for mixing of enzymes with their substrates or with the factors that might activate the

enzymes.

oxygenases found in its genome were directly involved in 1-octen-3-ol formation. A. fumigatus

Volatile analysis of oxygenase mutants of $A$. flavus suggested that none of the five

PpoC forms (R)-10-HPO from linoleic acid (Garscha et al., 2007) while $(R)$-1-octen-3-ol is

formed stereospecifically from $(S)$-10-HPO in mushrooms (Agaricus bisporus) (Wurzenberger \&

Grosch, 1984). Even though product specificity of A. flavus PpoC has not been identified yet, the

discrepancy in stereochemistry further reduces the possibility that PpoC is involved in $(R)-1$ -

octen-3-ol formation in A. flavus. This is unexpected because these are the only genes showing

substantial homology to the fatty acid oxygenases involved in oxylipin pathways in animal and

plant cells. Because the ability to form 1-octen-3-ol after disruption of conidia was abolished by

heat-treatment, there must be another enzyme system(s) that is responsible for 1-octen-3-ol

formation. It is known that deletion or overexpression of a ppo gene can result in abberant

regulation of other ppo genes through feedback regulation. For example, overexpression of $p p o A$

in $A$. nidulans led to reduced expression of $p p o C$, and deletion of $p p o B$ increased expression of

212 рроС (Brown et al., 2009; Tsitsigiannis et al, 2004). Possibly some unknown feedback

213 mechanism had impact on the volatile production as assessed in this study. 
215 densities, we found that availability of glucose was an important factor as addition of glucose

216 could partially remediate the germination defect. We also found that a heat-labile component in

217 the media recovered from A. flavus conidia suspension incubated for $9 \mathrm{~h}$ at high density $(1.0 \mathrm{x}$

$21810^{9} \mathrm{~mL}^{-1}$ ) was partly accountable for inhibition of germination. However, in our set up, it did not

219 appear that 1-octen-3-ol was contributing to inhibition of germination. The maximum

220 concentration of 1 -octen-3-ol $-57.6 \mu \mathrm{M}$ after 10 cycles of freeze-thaw treatment of $1.0 \times 10^{9}$

221 spores $/ \mathrm{mL}^{-1}$ - was well under the $10 \mathrm{mM}$ of exogenous 1-octen-3-ol found to inhibit germination.

222 This is also the case with $A$. nidulans (Supplemental Fig. S1). From these results, it appears that

223 1-octen-3-ol is not the principal endogenous autoinhibitor responsible for inhibiting germination

224 at high spore density for either $A$. flavus or $A$. nidulans. In previous studies showing 1-octen-3-ol

225 suppression of germination of conidia, its amount formed from the conidia had not been

226 determined (Chitarra et al., 2004; Herrero-Garcia et al., 2011). In order to confirm the role of 1-

227 octen-3-ol in controlling germination of fungal conidia, the amount of 1-octen-3-ol formed by the

228 conidia should be precisely quantified, then, the effect of 1-octen-3-ol at the physiological

229 concentration should be re-evaluated. 
231 of their cell integrity. $(R)$-1-Octen-3-ol was most abundantly formed volatile. Analyses on mutant

232 strains revealed that fatty acid oxygenases identified in $A$. flavus so far, namely, ppoA, ppoB,

233 рроC, рроD, and lox were not essential to form 1-octen-3-ol. It is suggested that another, heat-

234 labile enzyme, is involved in 1-octen-3-ol biosynthesis. Even though increasing spore density

235 was correlated with germination inhibition for $A$. flavus, the amount of 1-octen-3-ol formed from

236 the conidia was much lower than that found to inhibit germination of conidia, thus, 1-octen-3-ol

237 is not directly involved in inhibition of germination and the physiological significance of 1-octen-

238 3-ol formation in conidia remains an open question.

\section{ADDITIONAL INFORMATION AND DECLARATIONS}

$240 \quad$ Funding

241 This work was supported in part by the Japan Society for the Promotion of Science (JSPS)

242 [KAKENHI (No. 23580151)] and by the Yamaguchi University (Yobimizu Project) to M.K., and

243 in part by NSF IOS-0965649 funds to N.P.K.

244 Grant Disclosures

245 Competing Interests

246 Nancy P. Keller is an Academic Editor for PeerJ. The other authors do not have any competing 
247 interests.

248 Author Contributions

$249 \cdot$ Kana Miyamoto conceived and designed the experiments, performed the experiments, analyzed

250 the data.

251 -Tomoko Murakami and Pattana Kakumyan performed the experiments.

252 -Nancy P. Keller supplied the specimen and wrote the paper.

253 Kenji Matsui conceived and designed the experiments, analyzed the data and wrote the paper.

254 Supplemental Information

255 Supplemental information for this article can be found online at $\mathrm{xxx}$. 


\section{REFERENCES}

257

258

259

260

261

262

263

264

265

266

267

268

269

270

271
Berendsen RL, Kalkhove SIC, Lugones LG, Baars JJP, Wösten HAB, Bakker PAHM. 2013.

Effect of the mushroom-volatile 1-octen-3-ol on dry bubble disease. Applied Microbiology

and Biotechnology 97:5535-5543.

Brodhun F, Feussner I. 2011. Oxylipins in fungi, FEBS Letters 278:1047-1063.

Brodhun F, Schneider S, Göbel C, Hornung E, Feussner I. 2010. PpoC from Aspergillus nidulans is a fusion protein with only one active haem. Biochemical Journal 425:553-565.

Brown SH, Scott JB, Bhaheetharan J, Sharpee WC, Milde L, Wilson RA, Keller NP. 2009. Oxygenase coordination is required for morphological transition and the host-fungus interaction of Aspergillus flavus. Molecular Plant-Microbe Interactions 22:882-894.

Chitarra GS, Abee T, Rombouts FM, Posthumus MA, Dijksterhuis J. 2004. Germination of Penicillium paneum conidia is regulated by 1-octen-3-ol, a volatile self-inhibitor. Applied and Environmental Microbiology 70:2823-2829.

Combet E, Henderson J, Eastwood DC, Burton KS. 2006. Eight-carbon volatiles in mushrooms and fungi: properties, analysis, and biosynthesis. Mycoscience 47:317-326.

Combet E, Henderson J, Eastwood DC, Burton KS. 2009. Influence of sporophore 
development, damage, storage, and tissue specificity on the enzymic formation of volatiles in mushrooms (Agaricus bisporus). Journal of Agricultural and Food Chemistry 57:37093717.

Gaff DF, Okong'o-Ogola O. 1971. The use of non-permeating pigments for testing the survival of cells. Journal of Experimental Botany 22:756-758.

Garscha U, Jernerén F, Chung D, Keller NP, Hamberg M, Oliw EH. 2007. Identification of dioxygenases required for Apergillus development. Studies of products, stereochemistry, and the reaction mechanism. Journal of Biological Chemistry 282:34707-34718.

Herrero-Garcia E, Garzia A, Cordobés S, Espeso EA, Ugalde U. 2011. 8-Carbon oxylipins inhibit germination and growth, and stimulate aerial conidiation in Aspergillus nidulans. Fungal Biology 115:393-400.

Inamdar AA, Hossain MM, Bernstein AI, Miller GE, Richardson JR, Bennett JW. 2013. Fungal-derived semiochemical 1-octen-3-ol disrupts dopamine packaging and causes neurodegeneration. Proceedings of National Academy of Sciences of the United States of America 110:19561-19566.

Kermasha S, Perraud X, Bisakowski B, Husson F. 2002. Production of flavor compounds by hydroperoxide lyase from enzymatic extract of Penicillium sp. Journal of Molecular 
Nemcovic M, Jakubikova L, Viden I, Farkas V. 2008. Induction of conidiation by endogenous volatile compounds in Trichoderma spp. FEMS Microbiology Letters 284:231-236.

Shimizu K, Keller NP. 2001. Genetic involvement of a cAMP-dependent protein kinase in a G protein signaling pathway regulating morphological and chemical transitions in Aspergillu nidulans. Genetics, 157:591-600.

Tsitsigiannis DI, Kowieski TM, Zarnowski R, Keller NP. 2004. Endogenous lipogenic regulators of spore balance in Aspergillus nidulans, Eukaryotic Cell 3:1398-1411.

Tsitsigiannis DI, Kowieski TM, Zarnowski R, Keller NP. 2005. Three putative oxylipin biosynthetic genes integrate sexual and asexual development in Aspergillus nidulans. Microbiology 151:1809-1821. isomer of linoleic acid by a hydroperoxide lyase in mushrooms (Psalliota bispora). 
304 Fig. 1. SPME-GC/MS analysis of volatiles formed from A. flavus conidia. The conidia prepared

305 from 1-week old GMM plates were suspended in GMM liquid medium to be $1.0 \times 10^{9}{\text { spores } \mathrm{mL}^{-}}^{-}$

$306^{1}$, and immediately (intact) or after one cycle of freeze-thaw treatment (freeze-thawed) the

307 volatiles were collected with SPME fiber at $22^{\circ} \mathrm{C}$, then, analyzed with GC-MS. Separation of the

308 enantiomers of 1-octen-3-ol is shown in the inset.

309 Fig. 2. Effect of freeze/thaw treatment on the amount of 1-octen-3-ol and on the integrity of conidia. (A) Conidia were collected from A. flavus grown for 1-week on GMM plates, and suspended in GMM liquid media to be $1.0 \times 10^{9}$ spores $\mathrm{mL}^{-1}$. They were frozen at $-20^{\circ} \mathrm{C}$ for 55 min and thawed at $30^{\circ} \mathrm{C}$ for $5 \mathrm{~min}$. After given cycles of freeze-thaw treatment, 1-octen-3-ol was extracted from the culture with $\mathrm{CHCl}_{3} /$ methanol and analyzed with $\mathrm{GC} / \mathrm{MS}$. The values are

314 means of three replicates, and error bars show standard error. Different letters indicate 315 statistically significant differences $(P<0.05)$ for the cycles determined by ANOVA (Tukey). (B) 316 The numbers of intensely and slightly stained conidia, and not-stained conidia after Evans Blue 317 staining were counted under microscope, and distribution was calculated. Different letters 
318 indicate statistically significant differences $(P<0.05$, Kruskal-Wallis, $n=300)$. The freeze-thaw-

319 treated conidia were incubated in $\mathrm{GMM}$ for $9 \mathrm{~h}$ at $29^{\circ} \mathrm{C}$, and their germination rates were

320 determined under microscope (shown in B with a line chart). Different letters on the symbols

321 indicate statistically significant differences $(P<0.05$, Kruskal-Wallis, $n=500)$. (C) Evans Blue

322 staining was performed with conidia served to freeze-thaw treatment for $0,1,3,5$, and 10 cycles.

323 Slightly stained and intensely stained conidia are pointed with open and closed triangles,

324 respectively.

325 Fig. 3. Germination of conidia and elongation of hyphae were suppressed under high conidial

326 density (A), and in the presence of high concentration of 1-octen-3-ol (B). (A) Conidia of $A$.

327 flavus were prepared from 1-week old GMM plates, then, resuspended in GMM at $1.0 \times 10^{6}$ to

$3281.0 \times 10^{9}$ spores $\mathrm{mL}^{-1}$. The suspensions were incubated at $29^{\circ} \mathrm{C}$ for $9 \mathrm{~h}$, then, the germination rate

329 and the length of hyphae of germinated conidia were examined under microscope. (B) To the

330 conidia set at $1.0 \times 10^{6}$ spores $\mathrm{mL}^{-1}$, 1-octen-3-ol was added. Germination of conidia was

331 examined as above. Different letters indicate statistically significant differences $(P<0.05$,

332 Kruskal-Wallis, $n=200)$. 
333 Fig. 4. Effect of glucose concentration on germination of $A$. flavus conidia. The conidia were 334 suspended with GMM liquid medium containing 0 to $4 \%$ glucose. After incubating at $29^{\circ} \mathrm{C}$ for $3358.5 \mathrm{~h}$, the germination of conidia was examined under microscope. Different letters indicate 336 statistically significant differences among respective density $(P<0.05$, Kruskal-Wallis, $n=300)$.

337 Fig. 5. Effect of a factor secreted from the conidia at high density. The medium recovered from 338 the conidial suspension incubated for $9 \mathrm{~h}$ at $29^{\circ} \mathrm{C}$ at the density of $1.0 \times 10^{9} \mathrm{~mL}^{-1}$ (spent medium), 339 the used medium after heat treatment at $100^{\circ} \mathrm{C}$ for $10 \mathrm{~min}$ (heat treated spent medium), or fresh 340 GMM medium was used to suspend conidia at $1.0 \times 10^{6} \mathrm{~mL}^{-1}$. Germination of conidia was examined after incubation at $29^{\circ} \mathrm{C}$ for $9 \mathrm{~h}$ as above. Different letters indicate statistically significant differences $(P<0.05$, Kruskal-Wallis, $n=200)$.

343 Table 1. The amounts of 1-octen-3-ol formed from the freeze-thaw-treated conidia of ppo and lox 344 mutants. 


\section{Table 1 (on next page)}

The amounts of 1-octen-3-ol formed from the freeze-thaw-treated conidia of ppo and lox mutants 
Table 1. The amounts of 1-octen-3-ol formed from the freeze-thaw-treated conidia of ppo and lox mutants.

\begin{tabular}{|c|c|}
\hline Strain & 1-Octen-3-ol $\left(\mathrm{nmol} \mathrm{ml}^{-1}\right)^{\mathrm{a}}$ \\
\hline WT & $28.5 \pm 6.48$ \\
\hline$\triangle p p o A$ & $39.4 \pm 8.74$ \\
\hline$\Delta p p o C$ & $34.1 \pm 7.13$ \\
\hline$\Delta p p o D$ & $44.2 \pm 13.07$ \\
\hline$\Delta l o x$ & $52.1 \pm 13.17$ \\
\hline IRT4 & $30.7 \pm 8.54$ \\
\hline
\end{tabular}

${ }^{a}$ The conidia were suspended with GMM to be $1.0 \times 10^{9} \mathrm{ml}^{-1}$, then, the amount of 1 octen-3-ol was determined after one cycle of freeze-thaw treatment. The mean $\pm \mathrm{SE}$ is shown $(n=9)$. There was no statistically significant difference in the amount of 1-octen3-ol between each strain (ANOVA, Tukey). 


\section{Figure 1}

SPME-GC/MS analysis of volatiles formed from $A$. flavus conidia.

The conidia prepared from 1-week old GMM plates were suspended in GMM liquid medium to be $1.0 \times 10^{9}$ spores $\mathrm{mL}^{-1}$, and immediately (intact) or after one cycle of freeze-thaw treatment (freeze-thawed) the volatiles were collected with SPME fiber at $22^{\circ} \mathrm{C}$, then, analyzed with GC-MS. Separation of the enantiomers of 1-octen-3-ol is shown in the inset.

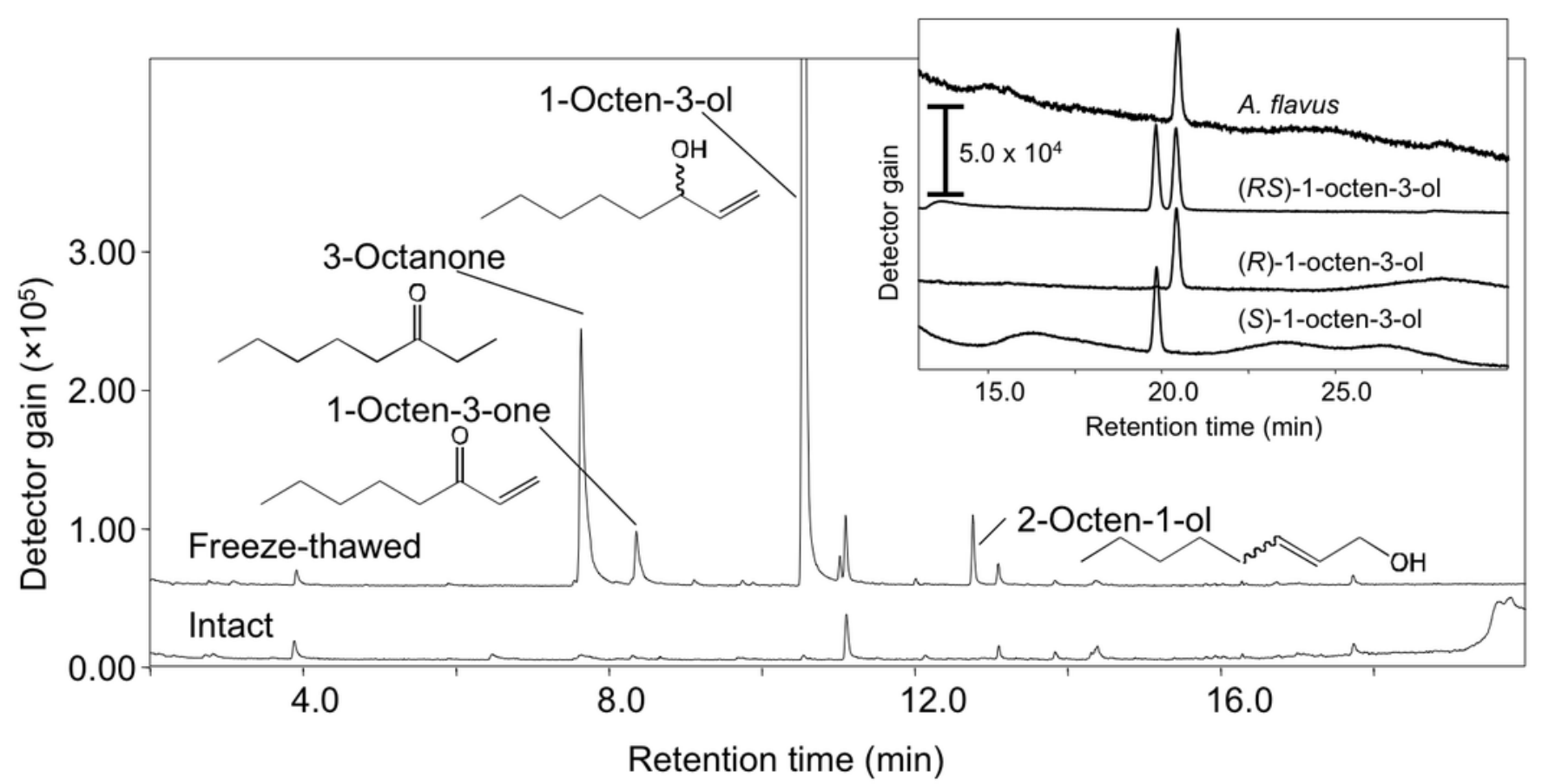




\section{Figure 2}

Effect of freeze/thaw treatment on the amount of 1-octen-3-ol and on the integrity of conidia.

(A) Conidia were collected from A. flavus grown for 1-week on GMM plates, and suspended in GMM liquid media to be $1.0 \times 10^{9}$ spores $\mathrm{mL}^{-1}$. They were frozen at $-20^{\circ} \mathrm{C}$ for $55 \mathrm{~min}$ and thawed at $30^{\circ} \mathrm{C}$ for $5 \mathrm{~min}$. After given cycles of freeze-thaw treatment, 1-octen-3-ol was extracted from the culture with $\mathrm{CHCl}_{3} /$ methanol and analyzed with $\mathrm{GC} / \mathrm{MS}$. The values are means of three replicates, and error bars show standard error. Different letters indicate statistically significant differences $(P<0.05)$ for the cycles determined by ANOVA (Tukey).

(B) The numbers of intensely and slightly stained conidia, and not-stained conidia after Evans Blue staining were counted under microscope, and distribution was calculated. Different letters indicate statistically significant differences $(P<0.05$, Kruskal-Wallis, $n=300)$. The freeze-thaw-treated conidia were incubated in GMM for $9 \mathrm{~h}$ at $29^{\circ} \mathrm{C}$, and their germination rates were determined under microscope (shown in B with a line chart). Different letters on the symbols indicate statistically significant differences $(P<0.05$, Kruskal-Wallis, $n=500)$. (C) Evans Blue staining was performed with conidia served to freeze-thaw treatment for $0,1,3$, 5, and 10 cycles. Slightly stained and intensely stained conidia are pointed with open and closed triangles, respectively. 


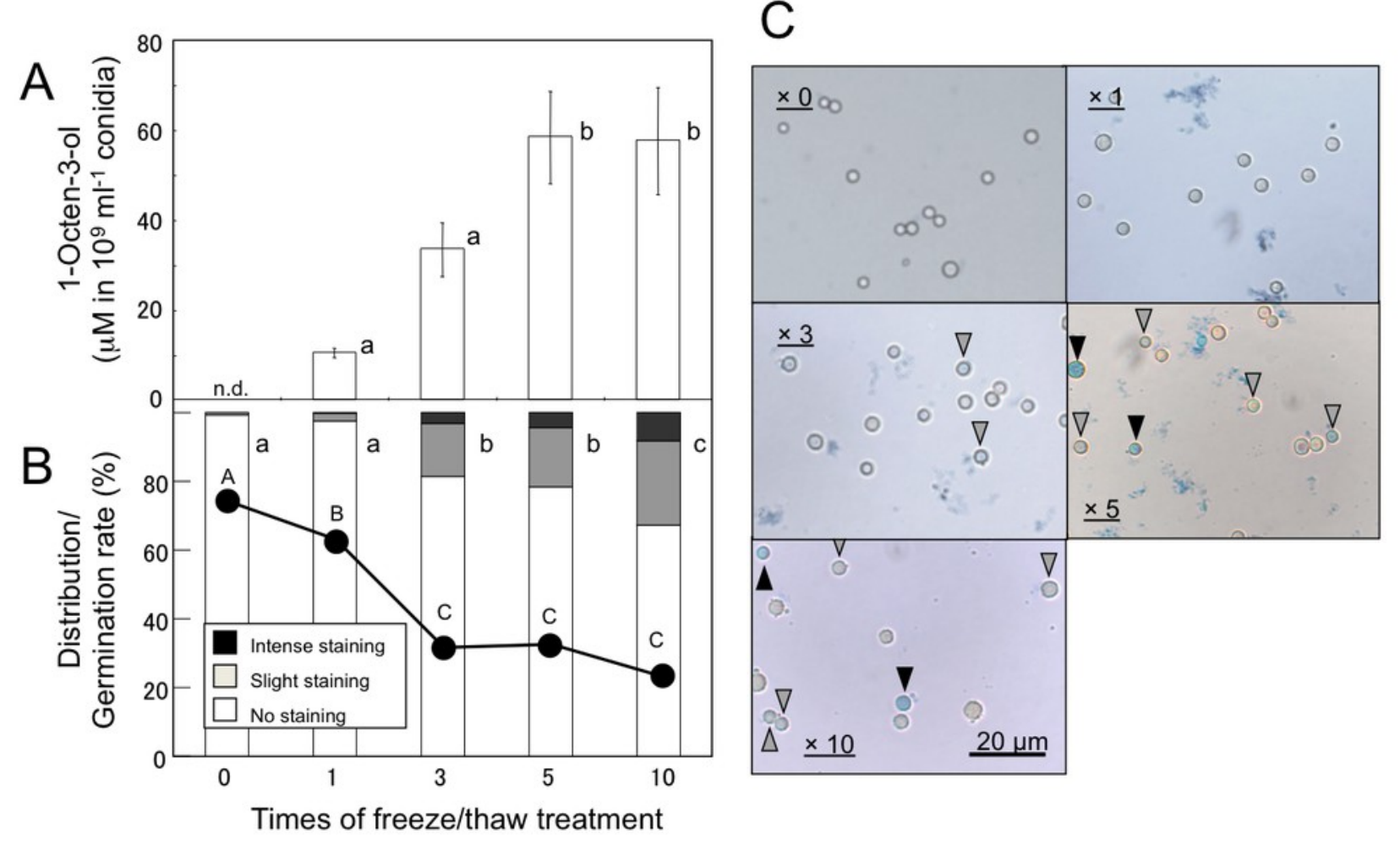




\section{Figure 3}

Germination of conidia and elongation of hyphae were suppressed under high conidial density $(A)$, and in the presence of high concentration of 1-octen-3-ol (B).

(A) Conidia of $A$. flavus were prepared from 1-week old GMM plates, then, resuspended in GMM at $1.0 \times 10^{6}$ to $1.0 \times 10^{9}$ spores $\mathrm{mL}^{-1}$. The suspensions were incubated at $29^{\circ} \mathrm{C}$ for $9 \mathrm{~h}$, then, the germination rate and the length of hyphae of germinated conidia were examined under microscope. (B) To the conidia set at $1.0 \times 10^{6}$ spores $\mathrm{mL}^{-1}, 1$-octen-3-ol was added. Germination of conidia was examined as above. Different letters indicate statistically significant differences $(P<0.05$, Kruskal-Wallis, $n=200)$.

A
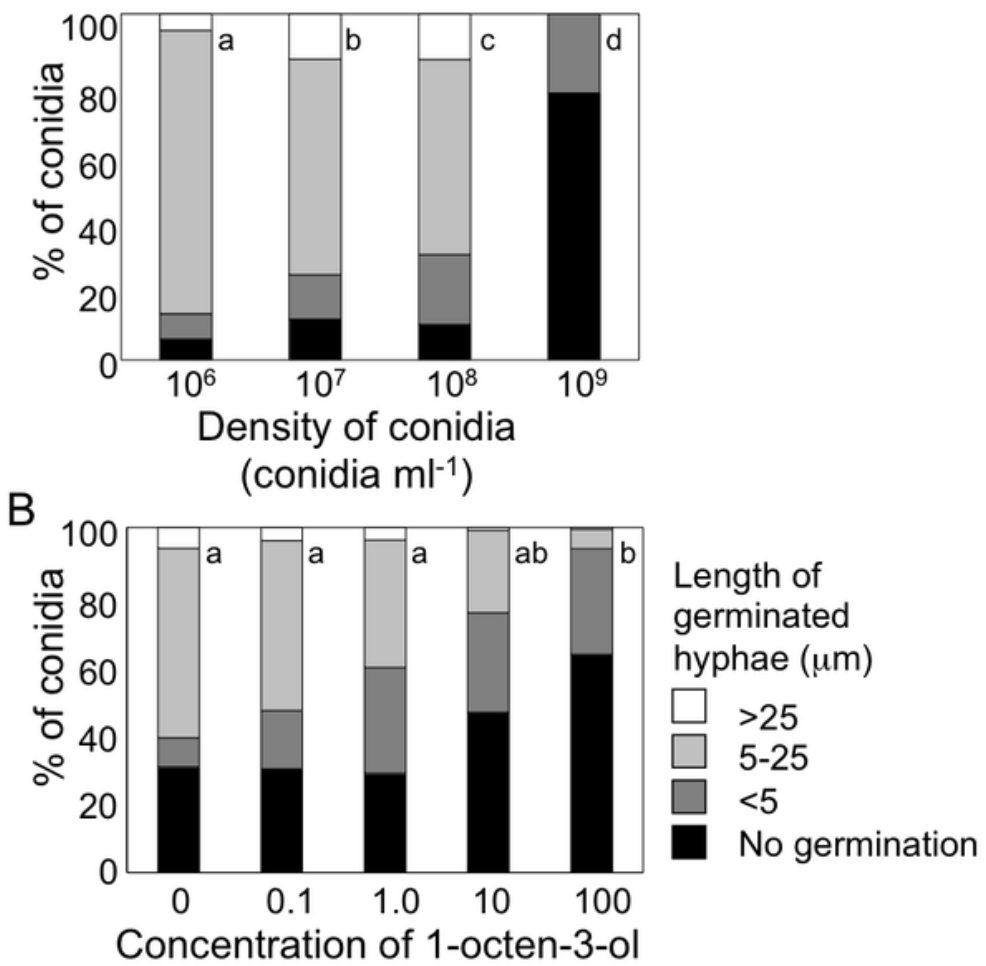

$(\mathrm{mM})$ 


\section{Figure 4}

Effect of glucose concentration on germination of $A$. flavus conidia.

The conidia were suspended with GMM liquid medium containing 0 to $4 \%$ glucose. After incubating at $29^{\circ} \mathrm{C}$ for $8.5 \mathrm{~h}$, the germination of conidia was examined under microscope. Different letters indicate statistically significant differences among respective density $(P<$ 0.05 , Kruskal-Wallis, $n=300$ ).

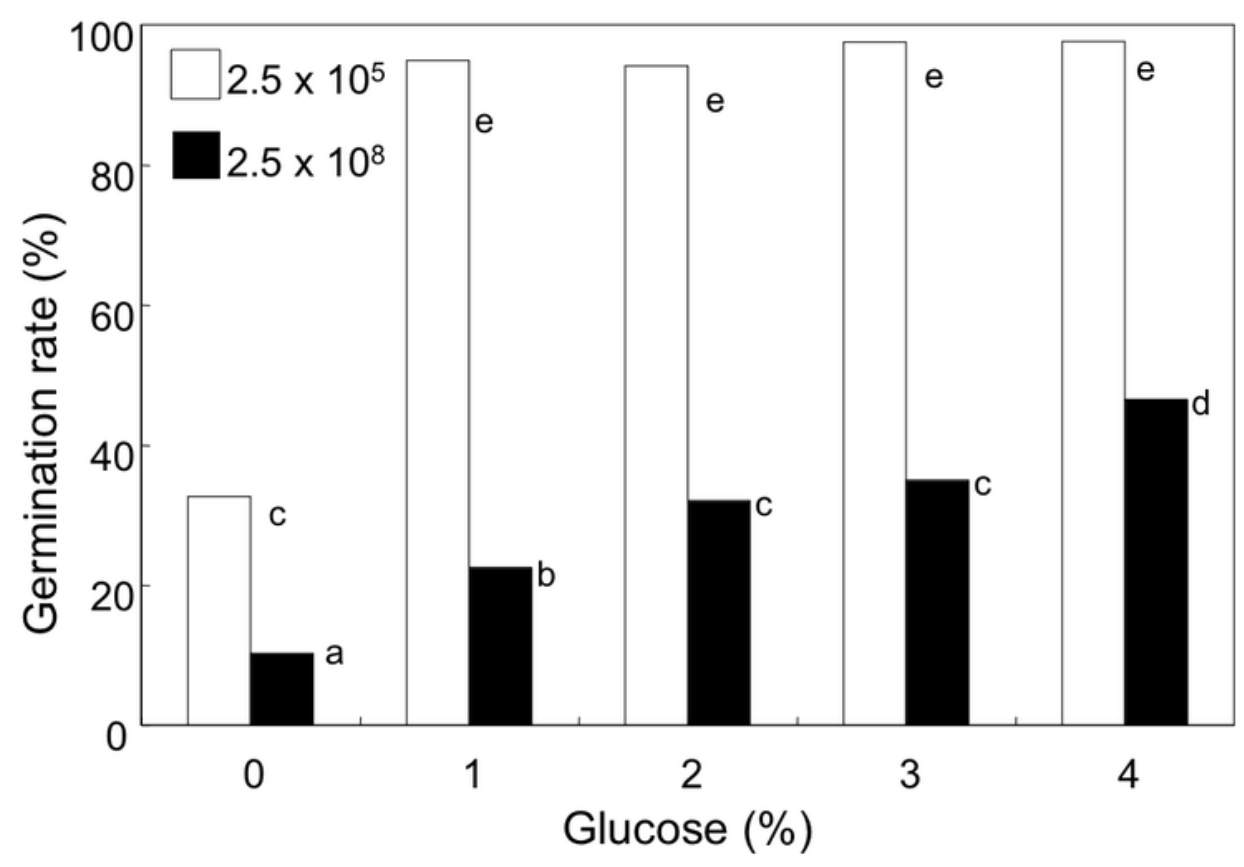




\section{Figure 5}

Effect of a factor secreted from the conidia at high density.

The medium recovered from the conidial suspension incubated for $9 \mathrm{~h}$ at $29^{\circ} \mathrm{C}$ at the density of $1.0 \times 10^{9} \mathrm{~mL}^{-1}$ (spent medium), the used medium after heat treatment at $100^{\circ} \mathrm{C}$ for $10 \mathrm{~min}$ (heat treated spent medium), or fresh GMM medium was used to suspend conidia at $1.0 \mathrm{x}$ $10^{6} \mathrm{~mL}^{-1}$. Germination of conidia was examined after incubation at $29^{\circ} \mathrm{C}$ for $9 \mathrm{~h}$ as above. Different letters indicate statistically significant differences $(P<0.05$, Kruskal-Wallis, $n=200)$.

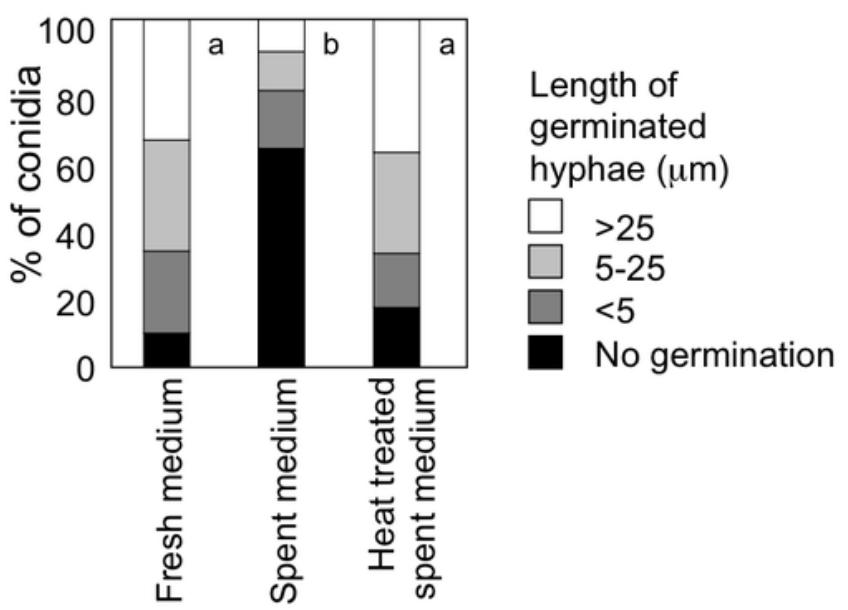

